\title{
Analysis of a Nonlinear First-Order System with a White Noise Input
}

\author{
T. K. Caughey and J. K. Difnes \\ California Institute of Technology, Pasadena, California
}

(Received June 19, 1961)

\begin{abstract}
A general technique for computing the power spectrum of nonlinear systems is presented, and the method is applied to the Brownian motion of a particle with idealized Coulomb damping. In this method the transition probability is obtained by solving the appropriate Fokker-Planck equation. Then the autocorrelation function is calculated by an appropriate integration, and finally the power spectrum is found by means of the Wiener-Khintchine relation. Comparison is made with the method of equivalent linearization, which is applicable to a wide class of nonlinear problems.
\end{abstract}

\section{OUTLINE OF THE TRANSITION PROBABILITY METHOD}

SYSTEMS which can be described by an ordinary $D$ differential equation and which are subject to random forces can be treated by the theory of continuous Markoff processes. Such processes are completely described if the transition probability, $T\left(q_{i}{ }^{0} \mid q_{i}, l\right)$, is known, where $i=1,2, \cdots, n$ and $n$ is the order of the system. The $q_{i}$ are the coordinates of the process and are frequently taken to be the system displacement and its first $n-1$ derivatives. The $q_{i}{ }^{0}$ are the initial values of these coordinates.

It is convenient to think of the transition probability as a density function in a space whose axes are the coordinates $q_{i}$. Then

$$
T\left(q_{i}{ }^{i} \mid q_{i}, l\right) \prod_{i=1}^{n} \Delta q_{i}
$$

is the probability that a system, which initially has coordinates $q_{i}{ }^{0}$, at a later time, $t$, has coordinates which lie in a volume element containing the point with coordinates $q_{i}$ and with sides of length $\Delta q_{i}$. The system coordinates must lie somewhere in this space, with probability one. Therefore,

$$
\int_{-\infty}^{\infty} \cdots \int_{-\infty}^{\infty} T\left(q_{i}{ }^{0} \mid q_{i}, t\right) \prod_{i=1}^{n} d q_{i}=1 .
$$

Furthermore, the system must have initially the coordinates $q_{i}{ }^{n}$. The transition probability must therefore satisfy the initial condition

$$
\lim _{t \rightarrow 0} T\left(q_{i}^{0} \mid q_{i}, t\right)=\delta\left(q_{1}-q_{1}^{0}\right) \delta\left(q_{2}-q_{2}^{0}\right) \cdots \delta\left(q_{n}-q_{n}^{0}\right) .
$$

The transition probability is the fundamental solution to the Fokker-Planck equation

$$
\dot{T}=-\sum_{i} \frac{\partial\left(A_{i} T\right)}{\partial q_{i}}+\frac{1}{2} \sum_{i, j} \frac{\partial^{2}\left(B_{i j} T\right)}{\partial q_{i} \partial q_{j}} .
$$

The coefficients $A_{i}$ and $B_{i j}$ are to be determined from the differential equations of the process, and are defined as follows:

$$
\begin{aligned}
\lim _{\Delta t \rightarrow 0}\left\langle\frac{\Delta q_{i}}{\Delta t}\right\rangle & =A_{i}, \\
\lim _{\Delta t \rightarrow 0}\left\langle\frac{\Delta q_{i} \Delta q_{j}}{\Delta t}\right\rangle & =B_{i j} .
\end{aligned}
$$

The derivation of the Fokker-Planck equation and the justification of the formulas for $A_{i}$ and $B_{i j}$ are given by Wang and Uhlenbeck. ${ }^{1}$

As the time $t$ goes to infinity, the dependence of the process on the initial conditions will disappear if the system approaches stationarity. The transition probability then approaches the first probability density, $W_{1}\left(q_{i}\right)$. This function satisfies the stationary FokkerPlanck equation.

$$
-\sum \frac{\partial\left(A_{i} W_{1}\right)}{\partial q_{i}}+\frac{1}{2} \sum_{i, j} \frac{\partial^{2}\left(B_{i j} W_{1}\right)}{\partial q_{i} \partial q_{j}}=0 .
$$

The second joint probability density, $W_{2}\left(q_{i}, q_{i}{ }^{10}, t\right)$, is the probability that the system has coordinates in the range $\left(q_{i}{ }^{0}, q_{i}{ }^{0}+\Delta q_{i}{ }^{0}\right)$ at time $l_{0}$ and in the range $\left(q_{i}, q_{i}+\Delta q_{i}\right)$ at time $t$. If the system is in a stationary state at time $t_{0}$ the second joint probability is the product of the first probability density at time $t_{0}, W_{1}\left(q_{i}{ }^{0}\right)$, and the transition probability density at a later time, $t, T\left(q_{i}^{0} \mid q_{i}, t\right)$. Then

$$
W_{2}\left(q_{i}{ }^{0}, q_{i}, t\right)=W_{\Upsilon}\left(q_{i}{ }^{0}\right) T\left(q_{i}{ }^{0} \mid q_{i}, t\right)=f^{1}{ }_{n}\left(t-t_{0}\right) .
$$

By means of the second probability density it is possible to calculate ensemble averages of functions which depend on the state of the system at two different times. The autocorrelation is such a function, and is a useful statistic in the analysis of random processes. It is defined as follows:

$$
\begin{gathered}
R_{j}(\tau)=\int_{\substack{-\infty \\
2 n \text {-fold }}}^{\infty} \cdots \int_{-\infty}^{\infty} W_{2}\left(q_{i}, q_{i}{ }^{0}, t\right) q_{j} q_{j}{ }^{0} \prod_{m=1}^{n} d q_{m}{ }^{0} \prod_{k=1}^{n} d q_{k} . \\
\tau=\left(t-l_{0}\right)
\end{gathered}
$$

It is convenient to define the mean value of the system

${ }^{1}$ M. C. Wang and G. E. Uhlenbeck, Revs. Modern Phys. 17, $323(1945)$. 
displacement $\left\langle q_{j}\left(q_{i}{ }^{0}, t\right)\right\rangle$ by the equation

$$
\left\langle q_{j}\left(q_{i}{ }^{0}, t\right)\right\rangle=\int_{-\infty}^{\infty} \cdots \int_{-\infty}^{\infty} q_{j} T\left(q_{i}{ }^{0} q_{i}, t\right) \prod_{k=1}^{n} d q_{k}=f^{\prime} n(\tau) .
$$

Then the autocorrelation can be written

$$
R_{j}(\tau)=\int_{-\infty}^{\infty} \int_{-\infty}^{\infty} q_{j}^{0}{ }^{0}\left\langle q_{j}\left(q_{i}^{0}, t\right)\right\rangle W_{1}\left(q_{i}{ }^{0}\right) \prod_{m=1}^{n} d q_{m^{0}} .
$$

It is sometimes useful to deal with the Laplace transform of the time dependent variables rather than with their explicit values. The Laplace transform will be denoted by a bar, that is

$$
\bar{f}(s)=\int_{0}^{\infty} e^{-8 \tau} f(\tau) d \tau
$$

Then (9) becomes

$$
\left\langle\bar{g}_{j}\left(q_{i}{ }^{0}, s\right)\right\rangle=\int_{-\infty}^{\infty} \cdots \int_{-\infty \text {-fold }}^{\infty} q_{j} \bar{T}\left(q_{i}{ }^{0} q_{i}, s\right) \prod_{k=1}^{n} d q_{k},
$$

and (10) can be written

$$
\bar{R}_{j}(s)=\int_{-\infty}^{\infty} \cdots \int_{-\infty}^{\infty} q_{j} W_{1}\left(q_{i}{ }^{0}\right)\left\langle\bar{q}_{j}\left(q_{i}{ }^{0}, s\right)\right\rangle \prod_{m=1}^{n} d q_{m} .
$$

The power spectrum and the autocorrelation are connected by the Wiener-Khintchine relation,

$$
\Phi_{j}(\omega)=\frac{2}{\pi} \int_{0}^{\infty} R_{j}(\tau) \cos \omega \tau d \tau
$$

Then the power spectrum can be obtained directly from the Laplace transform of the autocorrelation function by the formula below.

$$
\Phi_{j}(\omega)=(2 / \pi) \operatorname{Re} \bar{R}(i \omega),
$$

where Re means the "real part of." These relations demonstrate the connection between the theory of Markoff processes and the theory of power spectra and can, in principle, be used to determine the power spectrum of nonlinear systems.

\section{APPLICATION TO LINEAR SYSTEMS}

In order to illustrate the relations discussed above, the application to a linear first-order system is given. The governing equation of the process is

$$
\dot{y}+\beta y=N(l),
$$

where $N(t)$ is Gaussian and has a white power spectrum. This may be thought of as an equation for the velocity, $y$, of a mass with linear damping subject to a random force, $N(t)$. The appropriate Fokker-Planck equation is given in reference 1 .

$$
\dot{T}=\partial(\beta y T) / \partial y+D T_{y y} .
$$

Its fundamental solution is also given there, and is

where

$$
T=\left(2 \pi \sigma^{2}\right)^{-\frac{1}{2}} \exp \left[-\left(y-y_{0}{ }^{*}\right)^{2} / 2 .^{2}\right],
$$

$y_{0}{ }^{*}=\langle y\rangle=y_{0} \exp (-\beta l), \quad \sigma^{2}=D / \beta[1-\exp (-2 \beta t)]$.

The mean value, $\langle y\rangle$, of $y$ can be found as indicated in (9) by carrying out the integration, and is given above. The first probability density may be obtained by letting the time $t$ go to infinity.

$$
W_{1}(y)=(2 \pi D / \beta)^{-\frac{1}{2}} \exp \left(-\beta y^{2} / 2 D\right) .
$$

The autocorrelation of the velocity, $y$, is then found by substituting these results into (10).

$$
R(\tau)=D / \beta \exp (-\beta \tau) .
$$

Its Laplace transform is given by

$$
\bar{R}(s)=D / \beta(\beta+s) .
$$

Finally, the power spectrum is obtained using (14)

$$
\Phi(\omega)=\left(D / \pi / \beta^{2}+\omega^{2}\right)-\infty<\omega<\infty .
$$

This result is well known and is usually derived by the method of spectral analysis. However, the transition probability method is somewhat more general since it applies to nonlinear systems.

\section{APPLICATION TO A NONLINEAR SYSTEM}

If, in the problem discussed above, the linear damping $\beta y$ is replaced by a nonlinear one $f(y)$, the governing equation becomes

$$
\dot{y}+f(y)=N(t)
$$

and the appropriate Fokker-Planck equation is

$$
\dot{T}=\partial / \partial y[f(y) T]+D T_{y y} .
$$

This problem has been considered by Dienes ${ }^{2}$ in his doctoral thesis along with some higher order processes. MacDonald ${ }^{3}$ has also made some preliminary studies of the behavior of such systems.

The first probability density $W_{1}(y)$ is the solution to the stationary equation

$$
\frac{\partial}{\partial y}\left[f(y) W_{1}\right]+D \frac{\partial^{2} W_{1}}{\partial y^{2}}=0 .
$$

It is easily shown that the only solution of this equation

${ }^{2}$ J. K. Dienes, Ph.D. thesis, 1961, California Institute of Technology, Pasadena, California.

${ }^{3}$ D. K. C. MacDonald, Phys. Rev. 108, 541 (1957). 
satisfying the condition (1) is

$$
W_{1}=\frac{\exp [-F(y) / D]}{\int_{-\infty}^{\infty} \exp \left[\frac{-F(y)}{D}\right] d y},
$$

where $F(y)=\int f(y) d y$.

Although the first probability density can be determined for arbitrary damping, the transition probability cannot be so easily obtained. However, if the damping is of the idealized Coulomb type, the calculations outlined in the first section can be carried through explicitly. In this case $f(y)$ is of the form

$$
f(y)=k \operatorname{sgn} y \text {, }
$$

where sgny means the sign of $y$. Then the appropriate Fokker-Planck equation is

$$
\dot{T}=k \operatorname{sgn} y T_{y}+D T_{y y^{*}}
$$

Taking the Laplace transform and using the initial condition (2) an ordinary differential equation is obtained.

where

$$
D \bar{T}_{y y}+a \bar{T}_{y}-s \bar{T}=-\delta\left(y-y_{0}\right),
$$

$$
a=k \operatorname{sgn} y
$$

and is constant in the regions $y>0$ and $y<0$. With this notation, the general solution of $(29)$ is

$$
\begin{aligned}
& \widetilde{T}=A \exp \left\{\left[-a+\left(a^{2}+4 D s\right)^{\frac{1}{3}}\right] y / 2 D\right\} \\
& \quad+B \exp \left\{\left[-a-\left(a^{2}+4 D s\right)^{\frac{1}{}}\right] y / 2 D\right\} .
\end{aligned}
$$

The probability must vanish at $y= \pm \infty$. Furthermore, the effect of the delta function in (29) is the same as requiring a jump in $T_{y}$ at $y=y_{0}$; that is,

$$
\bar{T}_{y}\left(y_{0}+, s\right)-\bar{T}_{y}\left(y_{0}-, s\right)=1 / D .
$$

Therefore $\bar{T}(y)$ requires three different representations over the $y$ axis.

$$
\left.\begin{array}{ll}
\bar{T}=A \exp \left(\alpha_{1} y\right) & y \leqslant 0 \\
\bar{T}=B \exp \left(\alpha_{2} y\right)+C \exp \left(-\alpha_{1} y\right) & 0 \leqslant y \leqslant y_{0} \\
\bar{T}=E \exp \left(-\alpha_{1} y\right) & y>y_{0}
\end{array}\right\},
$$

where

$$
\alpha_{1}=\frac{\left(\lambda^{2}+s\right)^{\frac{1}{2}}+\lambda}{D^{\frac{1}{2}}}, \quad \alpha_{2}=\frac{\left(\lambda^{2}+s\right)^{\frac{1}{2}}-\lambda}{D^{\frac{1}{2}}}, \quad \lambda=\frac{k}{2 D^{1}} .
$$

There are four quantities, $A, B, C$, and $E$ to be determined, which are functions of $y_{0}$ and $s$, and these can be found from the four conditions: (a) the jump condition, (32); (b) continuity at $y=0$; (c) continuity at $y=y_{0}$; and (d) the transformed version of the normalization condition, (1)

$$
\int_{-\infty}^{\infty} \bar{T}\left(y, y_{0}, s\right) d y=1 / s .
$$

The result of solving the four simultaneous equations

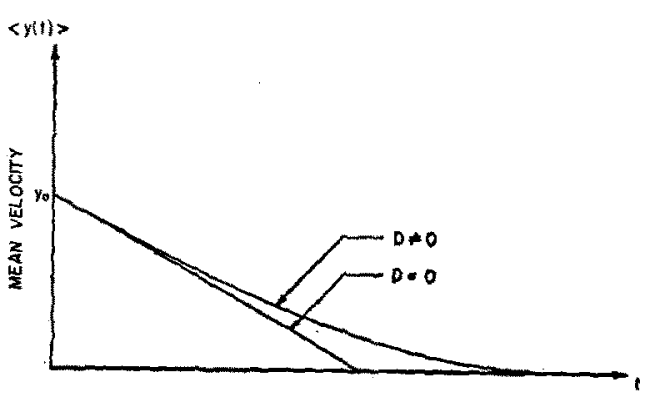

Fig. 1. The mean velocity of a mass with Coulomb damping subject to a random force with spectral density $2 D$.

arising from these conditions is given below.

$$
\left.\begin{array}{l}
A=\frac{\exp \left(-\alpha_{2} y_{0}\right)}{2 \alpha_{2} D} \quad B=\frac{\exp \left(-\alpha_{2} y_{0}\right)}{D\left(\alpha_{1}+\alpha_{2}\right)} \\
C=\frac{\alpha_{1}-\alpha_{2}}{\alpha_{1}+\alpha_{2}} \frac{\exp \left(-\alpha_{2} y_{0}\right)}{2 D \alpha_{2}} \\
E=\frac{\alpha_{1}-\alpha_{2}}{\alpha_{1}+\alpha_{2}} \frac{\exp \left(-\alpha_{2} y_{0}\right)}{2 D \alpha_{2}}+\frac{\exp \left(\alpha_{1} y_{0}\right)}{D\left(\alpha_{1}+\alpha_{2}\right)}
\end{array}\right\} .
$$

Equations (33), (34), and (35) together determine the Laplace transform of the transition probability explicitly. The inverse transform is given by

$$
\begin{aligned}
T=\frac{k \exp (-k|y| / D)}{2 \pi^{\frac{1}{2}} D} \int_{w}^{\infty} \exp \left(-u^{2}\right) d u \\
+\frac{\exp \left[k(y-|y|) / 2 D-\left(y-y_{0}+k t\right)^{2} / 4 D l\right]}{(4 \pi D t)^{\frac{1}{2}}}
\end{aligned}
$$

where

$$
w=\frac{y_{0}+|y|}{2(D t)^{\frac{3}{2}}} \text {. }
$$

The mean value of the velocity, given its initial value $y_{0}$, is difficult to evaluate explicitly, but its Laplace transform can be calculated from (11) by means of a straightforward, but lengthy, integration.

$$
\left\langle\tilde{y}\left(y_{0}, s\right)\right\rangle=\frac{y_{0}}{s}-\frac{k}{s^{2}}\left[1-\exp \left(-\alpha_{2} y_{0}\right)\right] .
$$

In the limit as $D$ goes to zero the solution to the deterministic initial value problem is recovered.

$$
\bar{y}(s)=y_{0} / s-k / s^{2} ; \quad y(t)=y_{0}-k t, \quad t \leqslant y_{0} / k
$$

In the absence of any imposed force, the velocity decreases linearly to zero, which is to be expected from elementary mechanics. The effect of the random force $N(t)$ is to make the decrease to zero velocity asymptotic as the time becomes infinite. This is illustrated in Fig. 1.

The Laplace transform of the autocorrelation $\bar{R}(s)$ can now be obtained by substitution of (38) into (12) and computing the first probability density $W_{1}(y)$ by 
combining (26) and (27).

$$
W_{1}(y)=(k / 2 D) \exp (-k|y| / D) \text {. }
$$

Then, upon carrying out the integration,

$$
\bar{R}(s)=\frac{D}{2 \lambda^{2} s}-\frac{D}{s^{2}}+\frac{4 \lambda^{2} D}{s^{4}}\left[\lambda-\left(\lambda^{2}+s\right)^{\frac{1}{2}}\right]^{2} .
$$

It may be noted here that the mean-squared displacement can be obtained from the above result by means of the Tauberian theorem

$$
\lim _{t \rightarrow 0} R(l)=\left\langle y^{2}\right\rangle=\lim _{s \rightarrow \infty} s \bar{R}(s)=\frac{2 D^{2}}{k^{2}} .
$$

Finally, the power spectrum is found by carrying out the algebra indicated in (14).

$$
\Phi(\omega)=\frac{D}{\pi \lambda^{4}}\left\{\frac{\lambda^{4}}{\omega^{2}}+\frac{8 \lambda^{8}}{\omega^{4}}\left[1-\left(\frac{1+\left(1+\omega^{2} / \lambda^{4}\right)^{\frac{1}{2}}}{2}\right)^{\frac{1}{2}}\right]\right\} .
$$

An ambiguity in the algebra arises because of the square root appearing in (41). The sign of the radical in (43) is chosen so that the power spectrum is finite at $\omega=0$, and its value there is $5 D^{3} / \pi k^{4}$.

It is convenient to deal with nondimensional power spectra. In order to accomplish this here, one can define a nondimensional frequency, $\eta=\omega / \lambda^{2}$, and a nondimensional spectrum $\phi(\eta)$ which is normalized to have a unit area.

$$
\phi(\eta)=2 \Phi(\omega) \lambda^{2} /\left\langle y^{2}\right\rangle, \quad 0<\eta<\infty .
$$

Then it follows that

$$
\int_{0}^{\infty} \phi(\eta) d \eta=1
$$

and the detailed expression for the normalized power spectrum is

$$
\left.\phi(\eta)=-\frac{4}{\pi} \mid \frac{1}{\eta^{2}}+\frac{8}{\eta^{4}}\left[1-\left(\frac{1+\left(1+\eta^{2}\right)^{\frac{1}{2}}}{2}\right)\right]\right\} .
$$

This function is plotted in Fig. 2, and is seen to be monotonically decreasing.

\section{THE EQUIVALENT LINEAR SYSTEM}

One motivation for the above analysis is that it provides a standard for evaluating the accuracy of the method of equivalent linearization, which provides a convenient approximation in many problems. Some applications are given in references 4 and 5 . For firstorder problems the linear system equivalent to $(23)$ is found by determining the value of $\beta$ which minimizes the expected mean-squared "deficiency" $\epsilon(y)$ in the equation

$$
\dot{y}+\beta y+\epsilon(y)=N(l),
$$

${ }^{4}$ T. K. Caughey, J. Appl. Mech. 26, 345 (1959).

${ }^{5}$ R. C. Booten, Jr., in Proceedings of the Symposium on Nonlinear Circuit Analysis (Polytechnic Institute, Brooklyn, New York, 1953), Vol. 2, pp. 369-391.

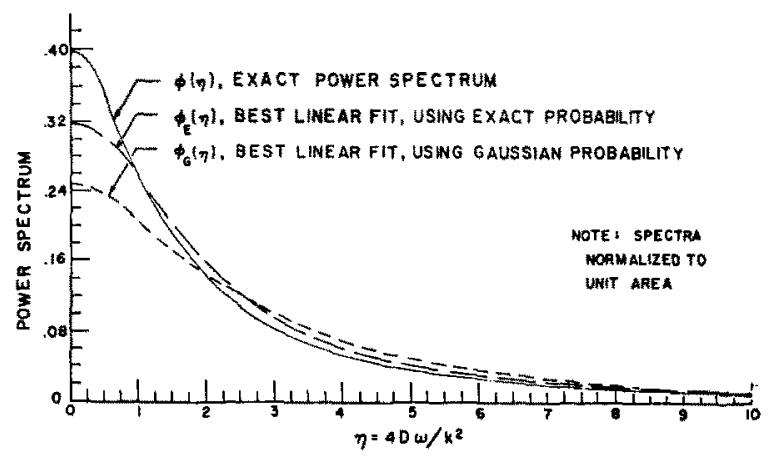

FIG. 2. Comparison of the exact and approximate power spectra of $y$, where $y+k \operatorname{sgn} y=N(t)$.

where

$$
\epsilon(y)=f(y)-\beta y .
$$

The expected value of the mean-squared deficiency is

$$
\left\langle\epsilon^{2}(y)\right\rangle=\int_{-\infty}^{\infty}[\beta y-f(y)]^{2} W_{1}(y) d y,
$$

and the value of $\beta$ which minimizes the expression above is

$$
\beta=\frac{\int_{-\infty}^{\infty} y f(y) W_{1}(y) d y}{\int_{-\infty}^{\infty} y^{2} W_{1}(y) d y} .
$$

The value of $\beta$ depends upon the choice of the first probability density. If the exact distribution (26) is used, the resultant value of $\beta$ will be called $\beta_{E}$. However, in many problems the exact first probability density is not known. In these cases it is frequently convenient to prescribe a Gaussian distribution. In this example, if a Gaussian distribution is chosen for $W_{1}(y)$ the equivalent damping is written $\beta_{G}$. Carrying out the integration for the example of Coulomb damping discussed above, the equivalent damping in the two cases is

$$
\beta_{E}=k^{2} / 2 D, \quad \beta_{G}=2 k^{2} / \pi D .
$$

Then the equivalent linear system is found by dropping the deficiency term $\epsilon(y)$ in (46)

$$
\dot{y}+\beta y=N(t) \text {. }
$$

Using the formula (22) the nondimensional power spectra of the equivalent systems are found.

$$
\phi_{E}=\left[\pi\left(1+\eta^{2} / 4\right)\right]^{-1} ; \quad \phi_{G}=\left[8\left(1+\pi^{2} \eta^{2} / 64\right)\right]^{-1} .
$$

The comparison of these two approximate spectra with the exact result is given in Fig. 2. It may be noted that for large $\eta$ the exact power spectrum $\phi(\eta)$ and the equivalent one $\phi_{E}(\eta)$ are both asymptotically equal to $4 / \pi \eta^{2}$, whereas the spectrum $\phi_{G}(\eta)$ is equal to $8 / \pi^{2} \eta^{2}$. The general agreement is somewhat better when the exact first probability density is used to minimize the deficiency than when the Gaussian distribution is used. 